\title{
Coding proposal on phenotyping heart failure with preserved ejection fraction: A practical tool for facilitating etiology-oriented therapy
}

\author{
Junbo $\mathrm{Ge}^{1,2}$ \\ ${ }^{1}$ Department of Cardiology, Zhongshan Hospital, Fudan University, Shanghai, China \\ ${ }^{2}$ Shanghai Institute of Cardiovascular Diseases, Shanghai, China
}

Editorial p. 6

With the publication of the PARAGON-HF (The Prospective Comparison of ARNI [angiotensin receptor-neprilysin inhibitor] with $\mathrm{ARB}$ [angiotensin-receptor blockers] Global Outcomes in Heart Failure with Preserved Ejection Fraction) trial during the European Society of Cardiology Congress 2019 [1], cardiologists again faced the reality that there remains no convincing evidenceproven strategy that can bring definitive benefit for heart failure (HF) with preserved ejection fraction (HFpEF). After hearing the news, many of the attendees' mood turned as blue as the sky over Paris. Certainly, we should keep moving forward, but before that, why not wait a minute and think about where the problem of $\mathrm{HFpEF}$ is.

To date, therapies tested in clinical trials on HFpEF have all been adopted from successful therapy concepts of heart failure with reduced ejection fraction (HFrEF). However, it seems to have been ignored that $\mathrm{HF}$ is a heterogeneous disease or syndrome, especially for HFpEF [2]. The heterogeneity in etiology and pathophysiology, alone or in combination, of $\mathrm{HFpEF}$ patients might explain why successful approaches in $\mathrm{HFrEF}$ patients failed however in HFpEF. One way to solve the HFpEF problem might be to go back to an etiology-oriented therapy principle and get rid of "one-size-fits-all" thinking mode. As early as 2012 , it was pointed out that $\mathrm{HFpEF}$ patients should be phenotyped according to etiology in order to make targeted therapies in $\mathrm{HFpEF}$ subgroups [2]. Shah et al. [3] once introduced a clinical phe- notypic classification of $\mathrm{HFpEF}$, which included: (1) "garden-variety" HFpEF; (2) coronary artery disease-HFpEF; (3) right heart failure-predominant $\mathrm{HFpEF}$; (4) atrial fibrillation-predominant $\mathrm{HFpEF}$; (5) hypertrophic cardiomyopathy-like HFpEF; (6) valvular HFpEF; (7) high output HFpEF; and (8) rare cause of HFpEF. Although this classification is oriented by the etiology and risk factors of $\mathrm{HFpEF}$, its intrinsic complexity has prevented its widespread clinical use. Hence, here I proposed a new coding method on phenotyping HFpEF, following principles of simplicity, practicality, and comprehensiveness.

The view expressed herein is that HFpEF can be classified into five subtypes (Table 1):

1. Vascular-related HFpEF. It includes HFpEF related to hypertension, coronary artery disease (CAD), and coronary microvascular dysfunction. Systemic and cardiac vascular abnormalities are both common in HFpEF, and that hypertension and $\mathrm{CAD}$ are considered the most prevalent cardiovascular comorbidities in HFpEF [4]. Furthermore, coronary microvascular dysfunction is also a common pathophysiological mechanism of HFpEF [4]. In these patients, hypertension or ischemia resulting from epicardial CAD or microvascular dysfunction plays a crucial role in the pathophysiology of HFpEF, so these patients might benefit from the HFrEF therapy strategy.

2. Cardiomyopathy-related HFpEF. These patients may have hypertrophic cardiomyopathy, infiltrative cardiomyopathies like cardiac amyloidosis and Fabry cardiomyopathy, etc. Diastolic dysfunction and elevation of left

Address for correspondence: Junbo Ge, MD, FACC, FESC, FSCAI, Professor of Medicine/Cardiology, Director, Department of Cardiology, Zhongshan Hospital, Fudan University, Chairman, Shanghai Institute of Cardiovascular Diseases, 180 Fenglin Road, 200032 Shanghai, China, e-mail: jbge@zs-hospital.sh.cn

Received: 18.12.2019 Accepted: 24.12.2019 
Table 1. New phenotypic coding for heart failure with preserved ejection fraction (HFpEF).

\begin{tabular}{|c|c|}
\hline Phenotypic coding & Brief description \\
\hline $\begin{array}{l}\text { HFpEF-1. Vascular- } \\
\text {-related HFpEF }\end{array}$ & $\begin{array}{l}\text { Hypertension, coronary artery disease, and coronary microvascular dysfunction } \\
\text { related HFpEF. }\end{array}$ \\
\hline $\begin{array}{l}\text { HFpEF-2. Cardiomyo- } \\
\text { pathy-related HFpEF }\end{array}$ & $\begin{array}{l}\text { These patients may have hypertrophic cardiomyopathy, infiltrative cardiomyopathies } \\
\text { like cardiac amyloidosis and Fabry cardiomyopathy which induced the HFpEF. }\end{array}$ \\
\hline $\begin{array}{l}\text { HFpEF-3. Right heart- } \\
\text { and pulmonary-related } \\
\text { HFpEF }\end{array}$ & $\begin{array}{l}\text { Patients in this subtype often have pulmonary hypertension with or without right } \\
\text { ventricular dysfunction. }\end{array}$ \\
\hline $\begin{array}{l}\text { HFpEF-4. Valvular- and } \\
\text { rhythm-related HFpEF }\end{array}$ & It mainly refers to HFpEF resulting from valvular disease and atrial fibrillation. \\
\hline $\begin{array}{l}\text { HFpEF-5. Extracardiac } \\
\text { disease-related HFpEF }\end{array}$ & $\begin{array}{l}\text { Extracardiac diseases involve: } \\
\text { (1) metabolic diseases, such as diabetes mellitus, obesity, or metabolic syndrome; } \\
\text { (2) diseases that often cause high output state, such as anemia, liver disease, } \\
\text { hyperthyroidism, and arteriovenous fistula; } \\
\text { (3) other diseases, such as chronic kidney disease, radiotherapy for cancer, etc. }\end{array}$ \\
\hline
\end{tabular}

ventricular filling pressure should be the underlying pathophysiology in these patients. Specific therapies, like targeted surgery or intervention in obstructive hypertrophic cardiomyopathy, tafamidis for transthyretin amyloidosis, enzyme replacement for Fabry should be considered in these cases.

3. Right heart- and pulmonary-related HFpEF. Patients in this subtype often have pulmonary hypertension with or without right ventricular dysfunction. Actually, pulmonary hypertension and right ventricular dysfunction could result from increased left ventricular filling pressure and left atrial hypertension, resulting in HFpEF in turn [3, 4]. Combined therapy from cardiologists and pulmonary specialists might be the key option for these patients.

4. Valvular- and rhythm-related HFpEF. Valvular diseases could lead to hemodynamic disorder in left and/or right heart, influencing the filling pressure and diastolic function further. Rhythm-related HFpEF mainly refers to $\mathrm{HFpEF}$ in which atrial fibrillation is predominant. Patients in this category might benefit from surgical or interventional valve therapy and rhythm control therapy.

5. Extracardiac disease-related HFpEF. The extracardiac diseases involve: (1) metabolic diseases, such as diabetes mellitus, obesity, or metabolic syndrome; (2) diseases that often cause a high output state, such as anemia, liver disease, hyperthyroidism, and arteriovenous fistula; (3) other diseases, such as chronic kidney disease, radiotherapy for cancer, etc. [5]. Again, etiology-oriented therapy options are needed for these patients, for instance, sodium-dependent glucose transporters 2 (SGLT2) inhibitor, weight-reduction efforts and exercise might be effective for metabolic HFpEF.

The new phenotype proposal for HFpEF is more structured than previously suggested [3]. The phenotypic coding not only helps introduce a better understanding about the risk factors, etiology, pathophysiology and clinical course of HFpEF, but also contributes to guide targeted treatment. The PARAGON-HF study confirmed again that, until now, no single therapy could treat $\mathrm{HFpEF}$ once and for all. Targeted treatment for etiologies and/or comorbidities may be the best choice. Proposed herein, it is high time to test the therapy principle by randomized clinical trials in $\mathrm{HFpEF}$ patients with identical or major etiologies according to the present coding proposal. It should be noted that an HFpEF patient could belong to one or more subtypes, and they can be defined as HFpEF-1, HFpEF-2, or a combination of HFpEF-13, HFpEF-135 for example.

\section{Conflict of interest None declared}

\section{References}

1. Solomon SD, McMurray JJV, Anand IS, et al. Angiotensin-Neprilysin Inhibition in Heart Failure with Preserved Ejection Fraction. N Engl J Med. 2019; 381(17): 1609-1620, doi: 10.1056/NEJMoa1908655, indexed in Pubmed: 31475794.

2. Shah AM, Pfeffer MA. The many faces of heart failure with preserved ejection fraction. Nat Rev Cardiol. 2012; 9(10): 555-556, doi: 10.1038/ nrcardio.2012.123, indexed in Pubmed: 22945329.

3. Shah SJ, Katz DH, Deo RC. Phenotypic spectrum of heart failure with preserved ejection fraction. Heart Fail Clin. 2014; 10(3): 407-418, doi: 10.1016/j.hfc.2014.04.008, indexed in Pubmed: 24975905.

4. Pfeffer MA, Shah AM, Borlaug BA. Heart failure with preserved ejection fraction in perspective. Circ Res. 2019; 124(11): 1598-1617, doi: 10.1161/CIRCRESAHA.119.313572, indexed in Pubmed: 31120821.

5. Saiki H, Petersen IA, Scott CG, et al. Risk of heart failure with preserved ejection fraction in older women after contemporary radiotherapy for breast cancer. Circulation. 2017; 135(15): 1388-1396, doi: 10.1161/CIRCULATIONAHA.116.025434, indexed in Pubmed: 28132957. 